\title{
Editorial
}

\section{La filosofía frente a la tecnología computacional digital o la invención de la digitalidad}

Jorge Francisco Maldonado Serrano

Universidad Industrial de Santander, Colombia 


\title{
La filosofía frente a la tecnología computacional digital o la invención de la digitalidad
}

\author{
Philosophy in front of digital computing \\ technology or the invention of digitality
}

Forma de citar (APA): Maldonado Serrano, J. F. (2020). La filosofía frente a la tecnología computacional digital o la invención de la digitalidad. Revista Filosofía UIS, 19(1), DOI: 10.18273/revfil.v19n1-2020014

Forma de citar (Harvard): Maldonado Serrano, J. F. (2020). La filosofía frente a la tecnología computacional digital o la invención de la digitalidad. Revista Filosofía UIS, 19(1), 11-20.

Fecha de recepción: 11 de septiembre de 2019

Fecha de aceptación: 18 de septiembre de 2019

Jorge Francisco Maldonado Serrano: colombiano. Doctor en Problemas del Pensar Filosófico. Profesor titular de la Escuela de Filosofía en la Universidad Industrial de Santander.

ORCID iD: orcid.org/0000-0002-7707-154X

Correo electrónico: jmaldona@uis.edu.co 
Editorial: La filosofía frente a la tecnología computacional digital o la invención de la digitalidad

\section{La filosofía frente a la tecnología computacional digital o la invención de la digitalidad}

La filosofía parece tener sí una nueva realidad: lo digital. ¿Qué entender por ello? Se ha hecho una diferenciación entre lo real y lo virtual, entre lo digital y lo analógico, o similares, para tratar de entender cómo plantear el problema. Sin embargo, no es unánime lo que se quiere decir con esas palabras, o a lo que se hace referencia. Consideramos que se puede hacer una distinción si se hacen las precisiones adecuadas.

\section{La diferencia entre digital y analógico en términos generales}

Paul Ceruzzi (2012) sugiere que la distinción entre digital y analógico surgió de manera casi fortuita. El profesor J. V. Atanasoff propuso la construcción de una máquina que utilizara tubos al vacío para hacer uso efectivo de un sistema aritmético binario que permitiera resolver ecuaciones lineales de manera rápida y librar del tedioso y largo procedimiento a los seres humanos. Ceruzzi escribe:

En una propuesta que Atanasoff escribió en 1940, describió una máquina que computaba a altas velocidades con tubos al vacío. Él señaló que tuvo en consideración técnicas "análogas" pero que las descartó y prefirió el cálculo directo [de operaciones lógicas] (tal vez este sea el origen del término moderno de "análogo" para referirse a la maquinaria computacional) (2012, p. 34).

En este sentido, esta expresión "análogas" usada por Atanasoff en su propuesta habría sido incorporada en el discurso computacional para diferenciar lo digital, como propuesta única. Las propuestas eran análogas porque usaban modelos matemáticos, pero no usaban sistemas aritméticos binarios. En contexto histórico, la oposición entre análogo y digital no hace referencia a algo propio de lo que es análogo sino todo lo que no es digital. Independiente de esta curiosidad histórica, podemos reconocer dos contextos en los que el uso de las expresiones permite entender mejor la diferencia entre digital y análogo.

El primer contexto es el de la información en general. Allí se habla de señal digital cuando se marcan unos límites para singularizar unidades discretas, con el fin de tomar algún tipo de medición, esto es, tomar información, de algo. Sin embargo, esta información o medición que se toma de ese algo, no expresa todo lo que ese algo implica, contiene o puede indicarnos. La señal analógica, por oposición a la señal digital, hace referencia a cuando la medición, en virtud de la señal, representa el continuum mismo de lo medido. La medición analógica contiene más información de ese algo que se mide, aunque es claro que en su 
medida no se incluye la cosa misma. Por ejemplo, un termómetro de mercurio nos permite entender mejor porque en este el cambio de temperatura, por sutil que sea, cambia la altura del metal líquido dentro del filamento tubular de vidrio (señal). Si bien no tenemos ojos tan agudos para distinguir más allá de los centígrados previamente marcados en el termómetro, sabemos que el mercurio puede estar más lejos o más cerca de, por ejemplo, 37.6 grados. Un termómetro digital, por el contrario, hará caso omiso de las variaciones entre 37.6 y 37.7 y presentará en la pantalla numérica (señal) uno u otro resultado. El termómetro analógico señala el continuum de la temperatura mientras que el digital solo señala un paquete discreto, que bien puede abarcar una infinitud de pequeñas diferencias de temperatura, cuando el límite entre una décima de grado y otro se supera ${ }^{1}$. En este sentido, digital sería un calificativo para información discreta, mientras que analógico lo sería para información continua.

Habría un segundo contexto, que se puede considerar previo al anterior, en el que la diferencia entre digital y analógico resulta relevante. Se trata de la problematización de los tipos de conocimiento. No pretendemos hacer una historia de la epistemología, porque sobre los tipos de conocimiento se ha discurrido desde que hay filosofía. En efecto, bien se asumiera que hay partes del alma, o niveles de conocimiento, unos más lejos otros más cerca de la divinidad o la percepción, pareciera que nunca hemos considerado que solo hay un tipo o nivel de conocimiento. Para el argumento de este texto, proponemos recurrir solo a Kant, porque su influencia en lo que hoy día es la investigación sobre el conocimiento, el pensamiento y la información es bastante alta. En la Crítica de la Razón Pura, Kant (2011) asumió la diferencia entre la sensibilidad y su conocimiento sensible, y el entendimiento y su conocimiento conceptual. Sensibilidad y entendimiento son modos del uso teórico de la razón. Una de las diferencias radica en que para la sensibilidad su contenido, la sensación, le es dada, es decir, la sensibilidad es pasiva respecto del conocimiento que recibe, que Kant mismo llama lo múltiple; pero el conocimiento del entendimiento es activo porque determina y da significado a lo que la sensibilidad recibe, a partir de la estructura conceptual. Creemos que es posible ver en esta diferencia un claro antecedente de la diferencia entre analógico y digital, con lo cual dejamos sentado que para la filosofía esta distinción no le es ajena, sino que pareciera le es bastante propia.

Ahora bien, podemos ver un desarrollo posterior de esta mirada sobre los dos tipos de conocimiento en un artículo seminal de Dretske, y en el que también encontramos una conexión entre ambos contextos, es decir entre los tipos de

\footnotetext{
${ }^{1}$ El ejemplo serviría también para destacar que aun en el termómetro analógico de mercurio, en la medida en que tiene unas marcas previas de medición (regla) en el vidrio, nos da información discreta y, por lo tanto, digital. Las marcas determinan unos límites, pero el filamento de mercurio fluye de manera continua por entre estas marcas digitales.
} 
Editorial: La filosofía frente a la tecnología computacional digital o la invención de la digitalidad

conocimiento y la información digital/analógica. Dretske, en Knowledge and the Flow of Information (1981), se expresa de la siguiente manera:

una señal (estructura, evento, estado) porta la información que s es $\mathrm{F}$ en forma digital si y solo si la señal no porta información adicional sobre $s$, ningún tipo de información que no se contenga ya en el hecho de que $\mathrm{F}$ sea s. Si la señal sí porta información adicional sobre s, información que no esté contenida en el hecho de que $\mathrm{F}$ sea $s$, entonces diré que la señal porta información de manera analógica (p. 137).

Lo interesante es que el objetivo de Dretske al proponer esta definición es comprender la diferencia entre la percepción sensorial, que sería de tipo analógica, y la capacidad que tenemos, como seres humanos, para referirnos a algo de cualquier experiencia sensorial conceptualmente en el lenguaje, que sería de tipo digital. Grosso modo, el lenguaje, cuando se usa para hablar de la experiencia sensorial, equivaldría a información digital, una especie de señal digital, por cuanto conceptualiza la información sensorial, mientras que la sensación captada por los sentidos sería analógica puesto que porta más información de la que se expresa en el lenguaje que se utiliza para describirla.

Nos parece claro que esta reflexión de Dretske explica la diada digital/ analógico. La distinción entre ambas expresiones es relevante, por cuanto nos permite diferenciar tipos de conocimiento o tipos de información. Pero esto significa que las expresiones digital y analógico representan cualidades de la realidad específica que llamamos información o conocimiento.

Retomemos, entonces, la diferencia entre la información digital y la analógica. Lo que hemos señalado apunta a que la señal de información analógica es más compleja o más densa que la señal de información digital, que es sencilla o simple. Igualmente, por lo que señalaba Dretske, incluso el mismo Kant, el conocimiento sensible es más rico, pero, si se quiere aprovechar más requiere ser expuesto en el lenguaje o requiere ser conceptualizado ${ }^{2}$. Dicho en otras palabras, la información analógica que se puede tener sobre algo siempre será más rica que la información digital que se tenga sobre eso mismo.

Con la descripción precedente de los contextos, el de la información y el de los tipos de conocimiento, a partir de los cuales se puede entender la diferencia entre analógico y digital, no pretendemos afirmar que el conocimiento y la información sean lo mismo, iguales o sinónimos. Pero elaborar esta distinción supondría otro ejercicio reflexivo que incluiría problemáticas de tipo político, de la historia de las ciencias y de la epistemología. En todo caso lo nuestro no es una

${ }^{2}$ Es claro que la exposición kantiana implica que siempre sucede dicha conceptualización, por lo menos en cierto grado, mientras que para Dretske el paso al lenguaje o al concepto no es necesario. 
confusión entre dos ámbitos, el filosófico y el computacional o informacional. Al contrario, el interés por el conocimiento es algo muy propio de las investigaciones filosóficas y psicológicas, por tradición y, recientemente, de la neurociencia, así como de la investigación tecnológica en computación. Sherry Turkle (1997) ha explicado que el intercambio de expresiones e ideas entre quienes estudian el pensamiento humano (filósofos, psicólogos y neurocientíficos) y quienes estudian cómo hacer máquinas que hagan algo parecido al pensamiento humano, ha sido parte del desarrollo de nuestras ideas sobre lo digital, trátese de computadores, Internet o similares:

hacia finales de los años sesenta el conductismo estaba en decadencia. Algunos psicólogos estaban deseosos de abrir la caja negra de la mente humana y hablar sobre los procesos que tenían lugar en su interior. El ordenador jugó un rol metafórico importante en la desaparición del conductismo. La verdadera existencia del ordenador y el lenguaje que lo rodea dieron soporte a una forma de pensamiento sobre la mente que minó el conductismo. Los científicos informáticos desarrollaron por necesidad un vocabulario para hablar de lo que ocurría en el interior de sus máquinas, los estados internos de sus sistemas. Y los investigadores en IA utilizaron libremente el lenguaje mentalista para hacer referencia a sus programas, hablaban de sus «pensamientos», «intenciones»y «metas». Si las nuevas mentes-máquina tenían estados internos, el sentido común sugería que las personas debían tenerlos también (Turkle, 1997, p. 162ss.).

En el fondo, siempre requerimos de algún tipo de metáforas o imágenes cuando necesitamos referirnos a nuevas producciones tecnológicas.

Ahora bien, la importancia de la diferencia no es una curiosidad filosófica. De hecho, la información que porta la señal digital tiene una gran ventaja sobre la información que porta una señal analógica. Digámoslo de manera abreviada: un sistema de información simple o sencilla, como la señal digital, facilitó la construcción de máquinas que hicieran los procesos de cálculo matemático, restringidos hasta entonces al ser humano y a su capacidad de pensar, de manera más rápida y precisa. Así, el desarrollo y perfeccionamiento de la información digital permitió la invención de máquinas que pudieran registrar, almacenar, recuperar, calcular y transmitir información con mayor facilidad y precisión. Dicho en otras palabras, un sistema digital de señales dio paso a un conjunto tecnológico que podía manipular fácilmente información en señales digitales.

Esta manipulación digital es algo interesante porque ya no se trataba de máquinas concretas que hicieran una cosa u otra, sino de una máquina universal, como proponía Turing (2004): una máquina que pudiera simular cualquier máquina. En efecto, al ser capaces de construir una máquina que computara digitalmente sistemas de ecuaciones, se podía llevar el formalismo matemático de cualquier máquina a la computadora digital. En este sentido, la tecnología 
Editorial: La filosofía frente a la tecnología computacional digital o la invención de la digitalidad

digital, gracias a que opera con señales discretas muy simples, tiene un potencial computacional que todavía no acabamos de predecir.

Así las cosas, la diferencia entre lo digital y lo analógico radica en la discontinuidad o en la continuidad, y en el tipo de manipulación que podemos hacer de la información; a partir de allí, se entiende la importancia o el potencial de la tecnología computacional digital frente a otros sistemas tecnológicos. Pero, respecto de la filosofía, es preciso ver que ambas, la computación digital y la filosofía siempre han estado involucradas, cada una a su manera con la misma problemática. Desde allí, se entiende que la propuesta de Luciando Floridi (2011) sea precisamente de una filosofía de la información.

Una vez hecho este sucinto análisis, que resulta bastante interesante, pero que en muchos aspectos deja discusiones pendientes, podemos preguntar: ¿qué le significa a la filosofía esta manipulación de la tecnología computacional digital? Volver la mirada sobre la manipulabilidad que lo digital ha abierto para el mundo de la vida, es crucial para entenderlo.

\section{La digitalidad en y de la filosofía}

Queremos señalar tres sentidos en que la filosofía se las ve con la tecnología computacional digital, con lo digital. Con ello no excluimos otras posibilidades de explicación filosófica como las que ya hay. Los dos primeros niveles son sencillos y hasta cierto punto obvios, pero el tercero busca plantear el problema propiamente dicho.

\section{1. El apoyo de la tecnología computacional digital para la filosofía}

La tecnología digital computacional influencia casi todas las dimensiones de la actividad filosófica. La escritura (con los procesadores de texto, diccionarios, correctores y similares), la lectura (con las bases de datos que ofrecen miles de artículos, libros en los diferentes formatos digitales, los correos electrónicos entre colegas y similares), eventos donde se reúnen expertos (con las video conferencias, proyecciones y demás), se realizan gracias a la tecnología computacional digital. Esta tecnología en filosofía nos da un acceso diferente, flexible y desjerarquizado porque la igualdad tiene una base efectiva $y$, a medida que el tiempo pasa, desaparecen los grandes secretos, los libros desconocidos, las obras de difícil acceso, etc.

Hemos dicho que en casi todas las dimensiones de la actividad filosófica porque pareciera que el acto mismo de pensar o de reflexionar o de criticar queda libre de dicha tecnología. En un sentido amplio, la salvedad tiene sentido. Pareciera que todo se media y regula ahora con la mencionada tecnología digital. Y resulta 
tan efectiva esta mediación que pasa completamente desapercibida y desaparece en lo que media. Esta obviedad nos conduce al segundo sentido precisamente porque la filosofía enfrenta lo obvio y lo cuestiona en su naturalidad.

\section{2. Lo digital como objeto de la filosofía}

El segundo sentido en que a la filosofía le interesa lo digital es como objeto o problemática de estudio. Consideramos que lo digital no solo puede ser objeto de estudio de la filosofía, sino que debe serlo. La aparición de las filosofías especiales, no solo puso en escena a la filosofía de la técnica y a la filosofía de la tecnología, sino a áreas específicas como la bioética, cuyas preguntas aumentan a medida en que la tecnología médica computarizada avanza.

Podemos considerar, además, varias áreas o especialidades filosóficas en que la filosofía se las ve con la tecnología computacional digital. En la medida en que los saberes actuales se han modificado, actualizado y desarrollado digitalmente, siempre la filosofía tendrá que considerar este giro digital en los saberes específicos. Las redes sociales empiezan a inquietar a la filosofía política, a la filosofía de la educación y a la filosofía del arte, solo por mencionar los más evidentes. Pero creemos que en todas las especialidades filosóficas la tecnología computacional digital cuestiona su forma de pensar y preguntar.

Y si la filosofía se las ve, como por ejemplo lo sugiere Clemént Rosset (2004), con el todo de la realidad, lo digital no solo hace parte de él, sino que ha transformado toda nuestra manera de concebir y de relacionarnos con la realidad entendida como totalidad. El hecho de que la filosofía siempre haya estado interesada por la realidad como totalidad, también hace que lo digital, en la medida en que permea ya hoy día la realidad de manera total, deba ser puesto bajo su lupa indagadora.

Precisamente los diferentes acercamientos a lo digital son impredecibles y funcionan, en este sentido como la filosofía: aunque podamos controlar algunos desarrollos de la tecnología digital, en realidad, nadie es dueño de lo digital, ni de su impacto o formas de apropiación cotidiana. Es como si tuviera una existencia propia, se hubiera abierto sus propias posibilidades. Pretender limitar lo digital es tanto como pretender limitar la filosofía. Pero puesto en una formulación más propositiva, la apertura de la tecnología hacia lo desconocido refleja la apertura de toda la filosofía, así como la imposibilidad de determinar cómo la tecnología refleja la imposibilidad de determinar no solo a la filosofía sino a cualquier cuestión que tenga que ver con los seres humanos. 
Editorial: La filosofía frente a la tecnología computacional digital o la invención de la digitalidad

\subsection{La digitalización de la filosofía: un ajuste en la imagen- pensamiento}

La cuestión más problemática sobre lo digital en la filosofía es la digitalización del pensamiento mismo. Dretske había concebido que lo conceptual sería, hasta cierto punto, equivalente a lo digital, por oposición a lo sensible, que lo sería a lo analógico. La cuestión es preguntarnos si la experiencia digital permite algo nuevo al pensamiento. No solo porque se trate, como decíamos en el segundo punto, de un nuevo objeto de estudio, una especie de moda filosófica, sino porque independiente de lo que se haga en filosofía, de su objeto, lo digital ha afectado directamente nuestra forma de pensar.

Esto quiere decir que no solo es el medio de la filosofía, o el medio de casi todas nuestras actividades humanas sino la forma misma que nuestro pensamiento estaría adquiriendo. Basta considerar el hecho de que sin universo digital no habría sido posible la crisis financiera. El desarrollo económico de los últimos años navega por el universo digital sin restricciones. Este, por ejemplo, es un caso en el que se muestra cómo lo digital abre nuevas posibilidades. Nuestra economía actual sería prácticamente imposible sin este universo digital que nos atraviesa.

A la filosofía, considero, le pasa lo mismo, pero en su medio propio. Por lo menos, podemos señalar dos aspectos importantes. Primero, la superación de las ideas de inconmensurabilidad entre los sistemas filosóficos. Segundo, la posibilidad de volver a pensar la filosofía en un continuum humano, no solo occidental sino terrestre.

Una de las ideas sobre los sistemas filosóficos ha sido, precisamente, que se considera que no puede haber compatibilidad entre sistemas filosóficos y que la comparación o superación entre ellos es solo una ilusión; esta es una de las insistencias de la filosofía de Deleuze (1997). Pero encontramos que la compatibilidad entre sistemas puede darse si aceptamos que lo digital establece una nueva imagen de lo que significa pensar respecto de la filosofía misma. No serían sistemas incomunicables entre sí, sino que, más bien, serían como programas que se ejecutan en una máquina universal. Esta metáfora de la máquina universal, muy discutible, por cierto, nos permite dar un paso respecto de la manera en que se pregunta por la esencia de la filosofía. De preocuparnos por saber a priori qué es lo filosófico, podemos interesarnos por probar nuevas formas de hacer filosofía. Se trataría, entonces, de aceptar un proceso de experimentación que permitiría validar o rechazar ideas, conceptos o explicaciones en el campo filosófico.

La otra idea sobre la filosofía, derivada de esta idea de los sistemas que garantizarían una especificidad filosófica, es que esta tiene límites que pueden establecerse históricamente (Grecia) y geográficamente (Occidente). La tecnología 
digital computacional no solo ha permeado a todo el planeta y a todas las esferas, sino que ha elevado a un verdadero plano de igualdad a todos los seres humanos independiente de su tiempo y de su ubicación. Encontramos que la universalidad de la filosofía no radica en un tipo de razonamiento o de preguntas sino en el hecho mismo de preguntar. Una vez más, esta metáfora, también muy discutible, nos permite dar un paso respecto de la manera en que reconocemos a los otros como filósofos.

\section{A manera de conclusión: preguntas}

¿Hemos de tomar partido a favor o en contra de la tecnología computacional digital? ¿Hemos de aprovecharla a ciegas? ¿Hemos de ponerle límites? ¿Hemos de seguir el proyecto moderno de la crítica y seguir abiertos a lo que todavía no sabemos que puede en efecto resultar sin por ello dejar que suceda cualquier cosa fortuitamente? Creemos que antes que cerrarnos a lo digital, la filosofía encuentra un nuevo orden con el que puede seguir dialogando.

\section{Referencias}

Ceruzzi, P. E. (2012). Computing a Concise History. Cambridge: MIT Press.

Deleuze, G. y Guattari, F. (1997). ¿Qué es la filosofía? (T. Kauf, trad.). Barcelona: Editorial Anagrama.

Dretske, F. I. (1981). Knowledge and the Flow of Information. Massachusetts: MIT Press.

Floridi, L. (2011). The philosophy of information. New York: Oxford University Press.

Kant, I. (2011). Crítica de la Razón Pura. (M. Caimi, trad.). Ciudad de México: Fondo de Cultura Económica.

Rosset, C. (2004). Lo real: Tratado de la idiotez. (R. del Hierro Oliva, trad.). Valencia: Pre-Textos.

Turing, A. (2004). On Computable Numbers, with an Application to the Entscheidungsproblem (1936). En The Essential Turing: Seminal Writings in Computing, Logic, Philosophy, Artificial Intelligence, and Artificial Life: Plus The Secrets of Enigma (pp. 58-90). Oxford: Oxford University Press.

Turkle, S. (1997). La vida en la pantalla: La construcción de la identidad en la era de internet. (L. Trafí, trad.). Barcelona: Paidós. 